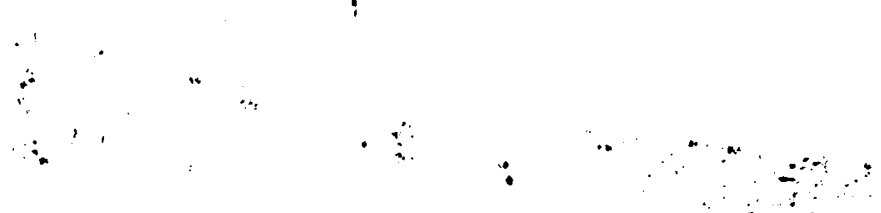

\title{
Letter to Editor: Luteinizing Releasing Factor and Müllerian-Inhibiting Substance: A Mistake?
}

\author{
-NATHALIE JOSSO \\ Unité de Recherches de Génétique Médicale, Hopital des Enfants Malades, 149, rue de Sères, 75730 Paris Cedex 15 \\ France
}

\begin{abstract}
I was very much intrigued by a paper by Bercu et al. recently published in Pediatric Research $(12: 139,1978)$. The authors claim to have demonstrated a significant increase in testicular Müllerianinhibiting activity of 17-day-old rat fetuses exposed to anti luteinizing hormone-releasing hormone serum in utero. In our experience, which confirms that of Picon (1970), this activity is already maximal in normal 17-day-old fetuses. Thinking that perhaps Holtzman rats used in Boston differed from the Wistar strain we work with, I turned to the paper by Donahoe cited by Bercu et al. (J. Surg. Res., 23: 141-148, 1977). There, I read that complete regression (5+) was observed from 14-21 days in a total of 95 experiments!
\end{abstract}

The conclusions reached by Bercu et al. are derived from an unstated number of observations (the number of male fetuses in two litters). The Student's $t$ test they use is appropriate only for quantitative, normally distributed data. Qualitative observations, such as those yielded by a graded bioassay, should be analyzed by a nonparametric method; for instance, the rank test of Wilcoxon or Whitney or Bross' (1958) "ridit method," which require much larger differences between groups to reach significance.

The attractive hypothesis put forward by Bercu et al. is not substantiated by the data published in Pediatric Research.

\section{Letter to Editor: Gonadotropin Control of Müllerian-Inhibiting Substance}

\section{BARRY B. BERCU, YASUHIDE MORIKAWA, IVOR M. D. JACKSON, AND PATRICIA K. DONAHOE}

The difference in the Müllerian-inhibiting substance activity in 17-day-old fetal rats in this experiment compared with previous observations could be due to several factors. In this report, we assayed fetuses from dams who had been handled daily; furthermore, the control dams were injected with normal rabbit serum. In addition, the activity of Müllerian-inhibiting substance in the testes of 6-day pups in luteinizing hormone-releasing hormone antiserum treated differed from that of the control groups, lending further support to the hypothesis that luteinizing hormone-releasing hormone and gonadotropins may regulate Müllerian-inhibiting substance secretion.

As stated in Table 1 of our published article, each mean represents tests from five to nine pups. In addition, we have since analyzed these data with Wilcoxon rank order statistical analyses and the significance remains unchanged. Our hypothesis is further substantiated by the observation that follicle-stimulating hormone inhibits Müllerian-inhibiting substance secretion in the luteinizing hormone releasing hormone-treated animals (1).

REFERENCES AND NOTES

1. Bercu, B. B., Morikawa, Y., Jackson, I. M. D., and Donahoe, P. K.: Inhibition of Müllerian inhibiting substance secretions by FSH. Pediatr. Res. (in press). 